This is a self-archived version of an original article. This version may differ from the original in pagination and typographic details.

Author(s): Khan, Wali Ullah; Jameel, Furqan; Ristaniemi, Tapani; Elhalawany, Basem M.; Liu,

Title: Efficient power allocation for multi-cell uplink NOMA network

Year: 2019

Version: Accepted version (Final draft)

Copyright: @ 2019 IEEE

Rights: In Copyright

Rights url: http://rightsstatements.org/page/InC/1.0/?language=en

Please cite the original version:

Khan, W. U., Jameel, F., Ristaniemi, T., Elhalawany, B. M., \& Liu, J. (2019). Efficient power allocation for multi-cell uplink NOMA network. In Proceedings of 2019 IEEE 89th Vehicular Technology Conference (VTC Spring). IEEE. IEEE Vehicular Technology Conference.

https://doi.org/10.1109/VTCSpring.2019.8746316 


\title{
Efficient Power Allocation for Multi-Cell Uplink NOMA Network
}

\author{
Wali Ullah Khan ${ }^{1}$, Furqan Jameel ${ }^{2}$, Tapani Ristaniemi ${ }^{2}$, Basem M. Elhalawany ${ }^{3}$, Ju Liu ${ }^{1}$ \\ ${ }^{1}$ School of Information Science and Engineering, Shandong University, Qingdao 266237, China. \\ ${ }^{2}$ Faculty of Information Technology, University of Jyvaskyla, FI-40014 Jyvaskyla, Finland. \\ ${ }^{3}$ Benha University, Egypt.
}

\begin{abstract}
Digital technologies are rapidly shaping the modern concepts of urbanization. It is a key element of developing practical smart cities of the future. In fact, they are the catalyst for the increasing networking of all areas of life in a smart city. Recent development in the domain of communication technologies has opened new avenues to realize the concept of smart cities. One of such communication technology is non-orthogonal multiple access (NOMA) for future cellular communications. This article, therefore, focuses on the interference management of uplink cellular NOMA systems. Specifically, we propose a power optimization technique for NOMA to improve the sum-rate in a multi-cell environment. We also consider Nakagami-m faded links to analyze the applicability of our proposed scheme under various channel conditions. The simulation results show that the proposed NOMA approach outperforms conventional orthogonal multiple access (OMA) technique in the multi-cell uplink scenario.

Index Terms - Nakagami-m, Non-orthogonal multiple access (NOMA), Multi-cell, Power optimization, Smart city
\end{abstract}

\section{INTRODUCTION}

Wireless communication is rapidly shaping the future of expanded connectivity of billions of devices. The future smart cities are mainly driven by the seamless connectivity of wireless devices. Digital infrastructure and mobility solutions are some of the key indicators of future smart cities [1], [2]. Another focal point is a modernization of legacy cellular infrastructure. Due to this reason, a key element of many smart city approaches is the central role of information and communication technologies (ICT) [3]. The ICT plays a key role in the concepts for future urban development. But a smart city is not a technological entity. It is rather a social-economic-technical structure. The city planners and technology companies are not so enthusiastic about the possibility of social exchange, but rather dream of a fully automated and fully connected city. In this context, a key element is the communication infrastructure that is able to satisfy the demands of the users [4]. In this direction, it becomes necessary to avoid orthogonal utilization of resources and move towards non-orthogonality. Therefore, it is anticipated that non-orthogonal multiple access (NOMA) is going to be one of the key enablers of future cellular technologies [5], [6].

NOMA works by accommodating multiple users on the same frequency/ time resource by assigning different power levels to them. Some of the key techniques used at the transmitter and receiver ends are the superposition coding (SC), and successive interference cancellation (SIC), respectively. The SC is one of the fundamental building blocks of information encoding techniques of communication theory. The objective of SC is to transmit information simultaneously at the same time to several nodes by a single transmitter. In [7], [8], Bergmans, and Gallager theoretically demonstrated the capability of SC to approach the capacity of both Gaussian broadcast channel and general channel. Later, the SC technique was applied to other communication channels including the multiple access, relay, interference, and wiretap channels. The experimental performance of SC technique was first investigated by Vanka et al. [9] in software-defined network. On the other hand, SIC is a well known physical layer decoding technique, that allows a receiver to decode different user packets simultaneously coming from the transmitter [10]. According to the principle of SIC technique, a pair of users multiplexed on the same spectrum resource will schedule according to their channel strength. The user with good channel condition will apply SIC, decode the signal of the user with weak channel quality, remove it and then decode its own signal. The weak user does not apply SIC and decode the signal with the interfering signal of the strong user [11].

There are several advantages of exploiting nonorthogonality of frequency and time resources. In particular, NOMA in power domain multiplexing is capable of accommodating multiple users with different types of quality of service (QoS) requests over the same spectrum/time resource [3], [6]. Therefore, power-domain NOMA is a key candidate technology for the Internet of Thing (IoT) which connects a large number of nodes. By exploiting the power domain for user multiplexing, NOMA is able to accommodate more users to cope with network overload. Unlike orthogonal multiple access (OMA) where users are served over exclusively allocated spectrum resources, NOMA can utilize the bandwidth more efficiently by accommodating multiple users over the same spectrum/time resource [12]. Besides this, unlike conventional OMA system with grantbased transmission where each user first needs to send the scheduling request for a grant to the base station which causes a high transmission delay and signaling cost, NOMA does not need scheduling [13]. Therefore, the transmission delay and signaling overhead are reduced drastically in NOMA systems.

Although NOMA techniques are very useful for multiplexing signals, interference among users belonging to different cells can degrade the decoding performance. Different group- 
ing strategies have been proposed to address the increased interference [14]-[16]. For instance, the authors of [14] proposed an interference mitigation strategy by selecting two users with high channel-gain difference which has been proved to improve the NOMA system sum rate considerable. The same scenario was extended for the case when a number of users are required to be assigned to the same group [15]. In a similar manner, Liang et al. in [16] proposed to optimize the capacity of a NOMA with the help of matching theory algorithm. Game theocratic approaches have also gained much prominence to reduce the effect of interference in NOMA [17], [18]. In this domain, a suboptimal strategy for half-duplex cognitive NOMA systems was proposed by the authors of [17]. Similarly, a matching game was proposed by Di et al. in [18] to connect users and mitigate the interference. This approach not only maximized the total sum rate but also improved the user fairness of NOMA systems.

However, there still exist many challenges in terms of interference mitigation that obstruct the widespread adoption of NOMA techniques. Firstly, most of the studies consider only single cell scenario where the base station (BS) employs NOMA techniques to distribute resources among users. Second, fewer studies exist in NOMA that takes into account channel variations and the effect of interference beyond conventional Rayleigh faded channels. Finally, to the best of our knowledge, there is a gap in the literature for studying interference mitigation for uplink NOMA scenarios, where most of the literature concentrate on downlink scenarios. To fill this glaring gap in NOMA literature, we aim to develop a comprehensive case study of uplink NOMA system that optimizes the power allocation to manage interference in a multi-cell environment. To understand the impact of channel variations in uplink NOMA, we have considered Nakagami$\mathrm{m}$ faded wireless links across all cells. The studies show significant improvement in NOMA gain for the proposed power allocation scheme.

The remainder of the paper is organized as follows. Section II provides an overview of the system model and problem formulation. Section III discusses the proposed power optimization scheme. In Section IV, we present simulation results along with their discussion. Finally, Section V provides some concluding remarks and future research directions.

\section{System Model and Problem Formulation}

We consider an uplink NOMA-based transmission as shown in Fig. $1^{1}$, which consists of $M$ cells, where $\mathcal{B S}=\left\{\mathrm{BS}_{m} \mid m=\right.$ $1,2,3, \ldots M\}$, constitute a network under co-channel technique. In each cell, a BS is located at the center and intends to communicate with $N$ different NOMA users (NUs), where $\mathcal{N U}=\left\{\mathrm{NU}_{n} \mid n=1,2,3, \ldots N\right\}$. We assume that all nodes of the network including BSs and NUs are equipped with a single antenna. In the network, each BS has complete knowledge of its serving NUs. We consider full spectrum

\footnotetext{
${ }^{1}$ In figure 1, the interference between cell of $\mathrm{BS}_{2}$ and cell of $\mathrm{BS}_{M}$ is due to co-channel deployment. The co-channel interference also exists among other cells but all are omitted for the sake of clarity.
}

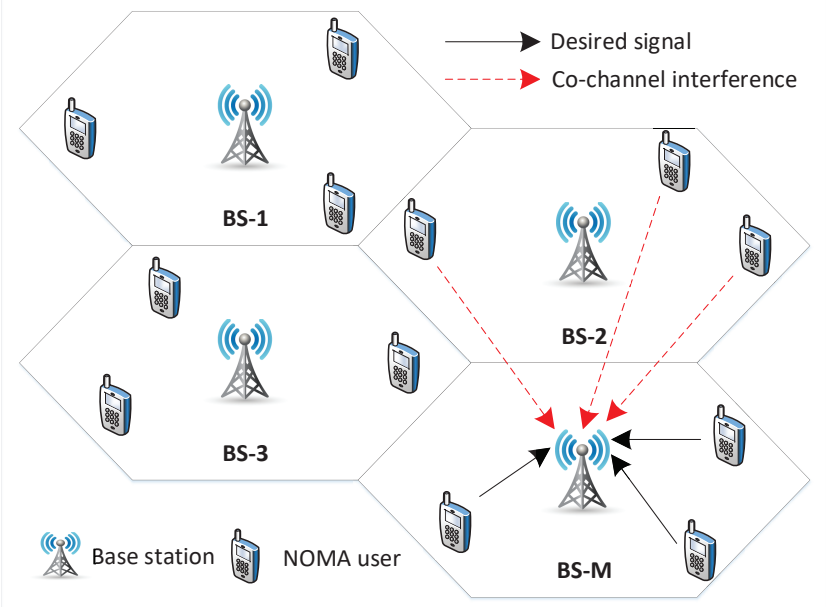

Fig. 1. Uplink transmission of multi-cell NOMA network

reuse such that each cell share the same spectrum resource and causes interference to other cells of the network. The channels between BSs and NUs undergo Nakagami fading. Without loss of generality, we sort the channel to co-channel interference plus noise ratios (CCINRs) of NUs associated with $\mathrm{BS}_{m}$ as

$$
\frac{h_{1, m}}{I_{1, m}^{\mathrm{CC}}+\sigma^{2}} \leq \cdots \leq \frac{h_{n, m}}{I_{n, m}^{\mathrm{CC}}+\sigma^{2}} \leq \cdots \leq \frac{h_{N, M}}{I_{N, M}^{\mathrm{CC}}+\sigma^{2}},
$$

where $h_{n, m}$ is the channel gain of $\mathrm{NU}_{n}$ at $\mathrm{BS}_{m}, \sigma^{2}$ is the variance of additive white Gaussian noise (AWGN), and $I_{n, m}^{\mathrm{CC}}$ is the co-channel interference from other cells to $\mathrm{BS}_{m}$ as

$$
I_{n, m}^{\mathrm{CC}}=\sum_{m^{\prime}=1, m^{\prime} \neq m}^{M} h_{m}^{n^{\prime}, m^{\prime}} \sum_{n^{\prime}=1, n^{\prime} \neq n}^{N} \chi_{n^{\prime}, m^{\prime}} p_{n^{\prime}, m^{\prime}}+\sigma^{2}
$$

To facilitate the uplink NOMA transmission, $\mathrm{BS}_{m}$ apply SIC to detect and decode multiple NUs signals. Let $s_{n, m}$ is a transmitted signal of $\mathrm{NU}_{n}$ to $\mathrm{BS}_{m}$, thereby the received signal of $\mathrm{NU}_{n}$ at $\mathrm{BS}_{m}$ can be expressed as

$$
\begin{aligned}
z_{n, m}= & \underbrace{h_{n, m} \chi_{n, m} \sqrt{p_{n, m}} s_{n, m}}_{\text {desired signal }}+\underbrace{\bar{\omega}}_{\text {noise }} \\
& +\underbrace{h_{n, m} \sum_{n^{\prime}=1, n^{\prime} \neq n}^{N} \chi_{n^{\prime}, m} \sqrt{p_{n^{\prime}, m}} s_{n^{\prime}, m}}_{\text {interference of NUs }} \\
& +\underbrace{\sum_{m^{\prime}=1, m^{\prime} \neq m}^{M} h_{m}^{n^{\prime}, m^{\prime}}\left(\sum_{n^{\prime}=1}^{N} \chi_{n^{\prime}, m^{\prime}} \sqrt{p_{n^{\prime}, m^{\prime}}} s_{n^{\prime}, m^{\prime}}\right)}_{\text {co-channel interference }},
\end{aligned}
$$

where $h_{n, m}$ is defined in (1), $\chi_{n, m}=1$ shows the $\mathrm{NU}_{n}$ association with $\mathrm{BS}_{m},{ }^{2} p_{n, m}$ is the $\mathrm{NU}_{n}$ transmit power, and $\bar{\omega}$ is the zero mean AWGN, respectively. According to the

\footnotetext{
${ }^{2}$ The users association to the BSs play an important role in enhancing performance of the network, however, is beyond the scope of this work.
} 
CCINRs order expressed in (1), the transmit power of NUs in $\mathrm{BS}_{m}$ should satisfy as

$$
p_{1, m}>\cdots>p_{n, m}>\cdots>p_{N, M} .
$$

Following (3), the received signal to interference plus noise ratio (SINR) of $\mathrm{NU}_{n}$ at $\mathrm{BS}_{m}$ is given by

$$
\tau_{n, m}=\frac{\chi_{n, m} p_{n, m}\left|h_{n, m}\right|^{2}}{I_{n, m}^{\mathrm{NU}}+I_{n, m}^{\mathrm{CC}}+\sigma^{2}},
$$

where $I_{n, m}^{\mathrm{NU}}$ is the interference of NUs after SIC process, and can be expressed as

$$
I_{n, m}^{\mathrm{NU}}=\left|h_{n, m}\right|^{2} \sum_{n^{\prime}=1, n^{\prime} \neq n}^{N} \chi_{n^{\prime}, m} p_{n^{\prime}, m} .
$$

We aim to minimize the co-channel interference and maximize the sum rate while guaranteeing the QoS of NUs in different cell. This can be obtained by solving the power optimization problem. Mathematically, the problem can be formulated as

$$
\begin{array}{ll} 
& \max _{p_{n, m}}\left\{\sum_{m=1}^{M} \sum_{n=1}^{N} R_{n, m}=\log _{2}\left(1+\tau_{n, m}\right)\right\} \\
\text { s.t. } & \sum_{m=1}^{M} R_{n, m} \geq \bar{R}_{m i n}, \quad \forall n, \\
& \sum_{m=1}^{M} \chi_{i, n} p_{n, m} \leq P_{m}, \quad \forall n, \\
& p_{n, m} \geq 0, \quad \forall n, m .
\end{array}
$$

where $\bar{R}_{\text {min }}$ and $P_{m}$ represent the minimum rate threshold for QoS and total battery power, respectively. Equation (7a) is the objective function of sum rate maximization. Constraint in (7b) guarantees the QoS of NUs in each cell, while constraints in (7c) and (7d) together limit the user transmit power, respectively.

\section{Proposed Power Optimization Scheme}

The objective of the optimization problem (7) is to mitigate the co-channel interference and maximize the sum rate of the system through optimal allocation of user transmit power at the uplink transmission of different cells. Due to co-channel interference, the optimization problem becomes non-convex which is hard to solve through traditional convex optimization scheme. Thus we provide a tractable suboptimal scheme where KKT conditions are satisfied. To do so, first we derive the Lagrangian function of optimization problem (7) as

$$
\begin{aligned}
\mathcal{L}(p, \phi, \psi)= & \sum_{m=1}^{M} \sum_{n=1}^{N} R_{n, m}+\sum_{n=1}^{N} \phi_{n}\left(\sum_{m=1}^{M} R_{n, m}-\bar{R}_{m i n}\right) \\
& \left.+\sum_{n=1}^{N} \psi_{n}\left(P_{m}-\sum_{n=1}^{M} p_{n, m}\right)\right),
\end{aligned}
$$

where $\phi_{n}$ and $\psi$ are the Lagrange multipliers. Applying KKT conditions as

$$
\begin{aligned}
\frac{\partial \mathcal{L}(.)}{\partial p_{n, m}}= & \frac{\partial}{\partial p_{n, m}}\left[\sum_{m=1}^{M} \sum_{n=1}^{N} R_{n, m}+\sum_{n=1}^{N} \phi_{n}\left(\sum_{m=1}^{M} R_{n, m}\right.\right. \\
& \left.\left.\left.-\bar{R}_{m i n}\right)+\sum_{n=1}^{N} \psi_{n}\left(P_{m}-\sum_{n=1}^{M} p_{n, m}\right)\right)\right], \\
\frac{\partial \mathcal{L}(.)}{\partial p_{n, m}}= & \frac{\partial}{\partial p_{n, m}}\left(\sum_{m=1}^{M} \sum_{n=1}^{N} R_{m, n}-\sum_{m=1}^{M} \phi_{n}\left(-\sum_{n=1}^{N} R_{n, m}\right)\right) \\
& -\psi_{n}, \\
\frac{\partial \mathcal{L}(.)}{\partial p_{n, m}}= & \frac{\partial}{\partial p_{n, m}}\left(\left(1+\phi_{n}\right) \log _{2}\left(1+\tau_{n, m}\right)+\sum_{i<n}^{N}\left(1+\phi_{i}\right)\right. \\
& \times \log _{2}\left(1+\tau_{i, m}\right)+\sum_{m^{\prime}=1, m^{\prime} \neq m}^{M} \sum_{n^{\prime}=1}^{N}\left(1+\phi_{n}^{\prime}\right) \\
& \left.\times \log _{2}\left(1+\tau_{n^{\prime}, m^{\prime}}\right)\right)-\psi_{n},
\end{aligned}
$$

$$
\begin{aligned}
& \frac{\partial \mathcal{L}(.)}{\partial p_{n, m}}=\left(1+\phi_{n}\right)\left(\frac{1}{\ln _{2}\left(1+\tau_{n, m}\right)} \times \frac{\partial}{\partial p_{n, m}}\left(1+\tau_{n, m}\right)\right) \\
& +\sum_{i=1, i \neq n}^{N}\left(1+\phi_{i}\right)\left(\frac{1}{\ln _{2}\left(1+\tau_{n, m}\right)} \times \frac{\partial}{\partial p_{n, m}}\left(1+\tau_{i, m}\right)\right) \\
& +\sum_{m^{\prime}=1, m^{\prime} \neq m}^{M} \sum_{n^{\prime}=1}^{N}\left(1+\phi_{n^{\prime}}\right)\left(\frac{1}{\ln _{2}\left(1+\tau_{n, m}\right)}\right. \\
& \left.\times \frac{\partial}{\partial p_{n, m}}\left(1+\tau_{n^{\prime}, m^{\prime}}\right)\right)-\psi_{n},
\end{aligned}
$$

$$
\begin{aligned}
\frac{\partial \mathcal{L}(.)}{\partial p_{n, m}}= & \left(1+\phi_{n}\right)\left(\frac{1}{\ln _{2}\left(1+\frac{p_{n, m}\left|h_{n, m}\right|^{2}}{I_{n, m}^{\mathrm{NU}}+I_{n, m}^{\mathrm{CC}}+\text { sigma }^{2}}\right)} \times \frac{\partial}{\partial p_{n, m}}\right. \\
& \left.\times\left(1+\frac{p_{n, m}\left|h_{n, m}\right|^{2}}{I_{n, m}^{\mathrm{NU}}+I_{n, m}^{\mathrm{CC}}+\sigma^{2}}\right)\right)+\sum_{i=1, i \neq n}^{N}\left(1+\phi_{i}\right) \\
& \times\left(\frac{1}{\ln _{2}\left(1+\tau_{n, m}\right)} \times \frac{\partial}{\partial p_{n, m}}\left(1+\tau_{i, m}\right)\right) \\
& +\sum_{m^{\prime}=1, m^{\prime} \neq m}^{M}\left(1+\phi_{n^{\prime}=1}\right)\left(\frac{1}{\ln _{2}\left(1+\tau_{n, m}\right)}\right. \\
& \left.\times \frac{\partial}{\partial p_{n, m}}\left(1+\tau_{n^{\prime}, m^{\prime}}\right)\right)-\psi_{n},
\end{aligned}
$$

After simplification, we obtain

$$
\begin{aligned}
\frac{\partial \mathcal{L}(.)}{\partial p_{n, m}}= & \left(1+\phi_{n}\right)\left(\frac{\lambda_{n, m}}{\ln _{2}\left(1+p_{n, m} \lambda_{n, m}\right)}\right) \\
& -\gamma_{n, m}^{\mathrm{NU}}-\gamma_{n, m}^{\mathrm{CC}}-\psi_{n}=0
\end{aligned}
$$

where $\lambda_{n}$ is the channel to interference plus noise ratio of $\mathrm{NU}_{n}$ associated with $\mathrm{BS}_{m}$, and can be given by

$$
\lambda_{n, m}=\frac{\chi_{n, m}\left|h_{n, m}\right|^{2}}{I_{n, m}^{\mathrm{NU}}+I_{n, m}^{\mathrm{CC}}+\sigma^{2}},
$$


while $\gamma^{\mathrm{NU}}$ and $\gamma^{\mathrm{CC}}$ are the interference of NUs after SIC process and co-channel interference causes by other cells, both are expressed as

$$
\begin{gathered}
\gamma_{n, m}^{\mathrm{NU}}=\sum_{i<n}^{M}\left(1+\phi_{i}\right)\left(\frac{\tau_{i, m}\left|h_{i, m}\right|^{2}}{\ln _{2}\left(1+\tau_{i, m}\right)\left(I_{i, m}^{\mathrm{NU}}+I_{i, m}^{\mathrm{CC}}+\sigma^{2}\right)}\right), \\
\gamma_{n, m}^{\mathrm{CC}}=\sum_{m^{\prime}=1, m^{\prime} \neq m}^{M} \sum_{n^{\prime}=1}^{N}\left(\frac{\left(1+\phi_{n^{\prime}}\right) \tau_{n^{\prime}, m^{\prime}}\left|h_{m}^{n, m}\right|^{2}}{\ln _{2}\left(1+\tau_{n^{\prime}, m^{\prime}}\right)\left(I_{n^{\prime}, m^{\prime}}^{\mathrm{NU}}+I_{n^{\prime}, m^{\prime}}^{\mathrm{CC}}+\sigma^{2}\right)}\right.
\end{gathered}
$$

For detailed derivation proof of (14), please refer to Appendix of [19]. The allocated power of $\mathrm{NU}_{n}$ associated with $\mathrm{BS}_{m}$ can be obtained from (14) as

$$
p_{n, m}^{*}=\left[\frac{\left(1+\phi_{n}\right)}{\gamma_{n, m}^{\mathrm{NU}}+\gamma_{n, m}^{\mathrm{CC}}+\psi_{n}}-\frac{1}{\lambda_{n, m}}\right]^{+},
$$

where $(\alpha)^{+}=\max (0, \alpha)$ and Lagrange multipliers can be obtained and iteratively updated by subgradient technique as

$$
\begin{gathered}
\phi_{n}(x+1)=\left[\phi_{n}(x)-\delta(x) \times\left(\sum_{m=1}^{M} \sum_{n=1}^{N} R_{n, m}-\bar{R}_{\text {min }}\right)\right]^{+}, \\
\psi_{n}(x+1)=\left[\psi_{n}(x)-\delta(x) \times\left(P_{m}-\sum_{m=1}^{M} \sum_{n=1}^{N} p_{n, m}\right)\right]^{+} \cdot(20)
\end{gathered}
$$

where $x$ index iteration number, and $\delta \geq 0$ is the step size. First, Lagrange multipliers $\phi_{n}$ and $\psi_{n}$ are updated using $p_{n, m}^{*}$ obtained from (18). Then, optimal $\phi_{n}$ and $\psi_{n}$ are used to calculate the optimal $p_{n, m}$. The iteration process can be ended on convergence.

Assuming the active number of NUs associated with each BS on the same spectrum resource is $N$. Then, the complexity of the solving problem (7) employing the proposed scheme in $x$ iteration is $\mathcal{O}\left(N^{2}\right)$. If the number of total iterations required for convergence of the proposed scheme is $K$, and the number of total BSs in the network is $M$, thereby the overall complexity of our proposed multi-cell model becomes $\mathcal{O}\left(K M N^{2}\right)$.

\section{Simulation Results}

In this section, we provide simulation results of the proposed power optimization technique. Unless mentioned otherwise, the initial user power is $3 \mathrm{~W}$ for each user, the number of active user in each cell is 3 and the number of cells varies from $1-7$.

Figure 2 shows the sum rate of the network against the increasing number of cells. It can be seen that the increase in the number of cells in the environment generally results in improving the sum rate. It is mainly due to the fact that the capability of accommodating new users also increases with an increase in the number of cells. To provide a fair comparison, we applied the proposed optimization scheme to both NOMA and OMA users. We noted that the power optimization strategy for NOMA outperforms the conventional OMA technique even for a single cell. Moreover, as the number of cells increases, the separation between OMA and

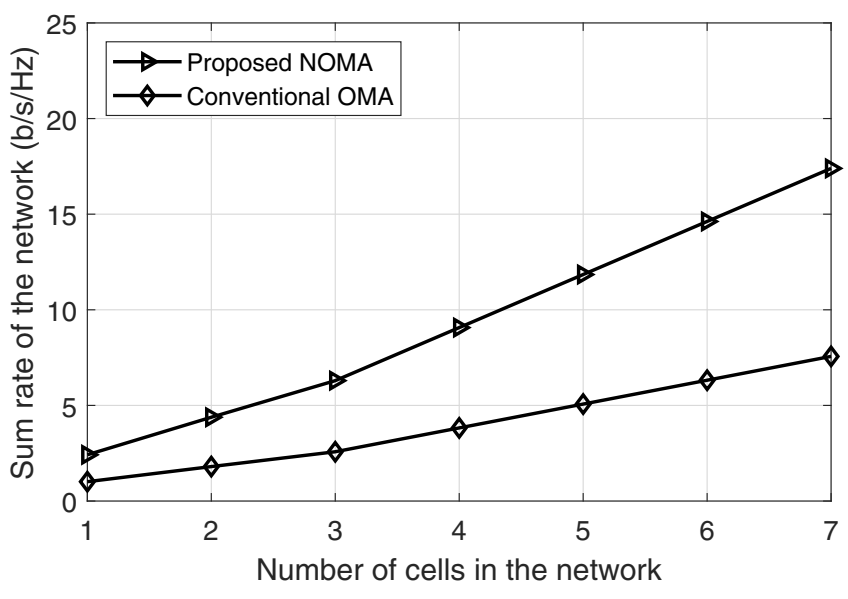

Fig. 2. Number of cells in the network versus sum rate of the network. The user transmit power is $3 \mathrm{~W}$, number of active user in each cell is 3 , and number of cells varies from 1-7.

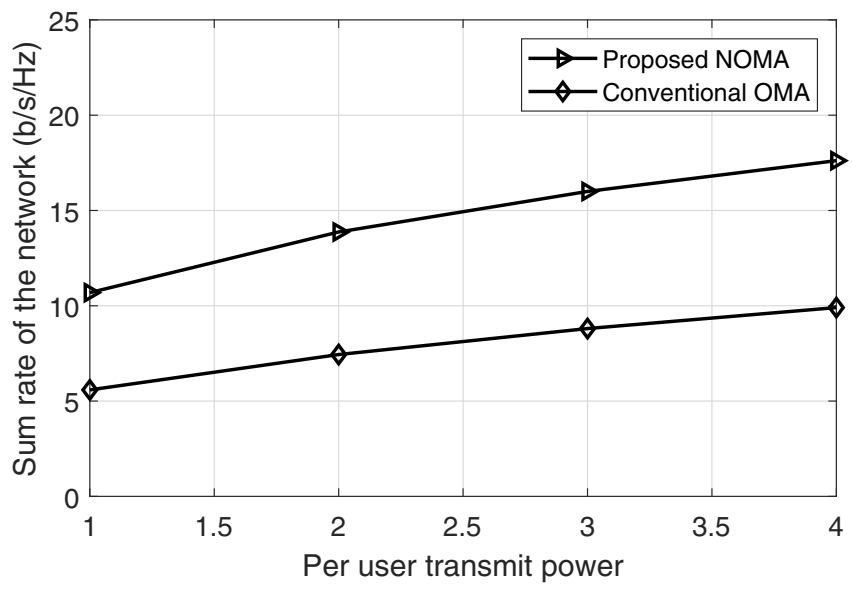

Fig. 3. Available user transmit power versus sum rate of the network. The number of cell is 7, number of active user in each cell is 3 , and power values for each user varies from 1-4 W.

NOMA grows. This indicates the effectiveness of our proposed strategy to manage interference as the number of cells grows. Specifically, when the total number of cells grow up to 7 , the OMA technique only achieves a sum-rate of $8 \mathrm{bit} / \mathrm{sec} / \mathrm{Hz}$, whereas, the sum-rate of proposed NOMA scheme becomes more than double by achieving $17 \mathrm{bit} / \mathrm{sec} / \mathrm{Hz}$.

Figure 3 shows the sum-rate as a function of per-user transmit power. One can observe that the sum-rate of the network increases with an increase in the transmit power of the user. However, similar to the last figure, as the transmit power of the individual user increases, the separation between OMA and proposed NOMA technique increases considerably. This also shows that the proposed NOMA technique is more flexible in managing interference against the increase in peruser power.

To highlight the impact of channel variations, we plot sumrate of the network against different values of Nakagami-m 